\section{A TELEPHONE REMINDER TO ENHANCE ADHERENCE TO INTERVENTIONS IN CARDIOVASCULAR RANDOMIZED TRIALS: A PROTOCOL FOR A STUDY WITHIN A TRIAL}

(SWAT)
Abstract

The impact of reduced adherence in randomized clinical trials is well documented in the 6 literature. Non-adherence can negatively affect the trial sample size and estimation of the 7 treatment effect. This study aims to evaluate the effects of a telephone call reminder on the 8 adherence rates of participants to interventions in a cardiovascular randomized trial. This is a 9 Study within a Trial (SWAT). The host trial is evaluating the effectiveness of a multidisciplinary 10 16-week cardiovascular disease prevention program on risk factor profile among patients with carotid artery stenosis. Simultaneously, this SWAT will evaluate the effectiveness of telephone call reminders on the participants' adherence to the host trial intervention. The primary outcome is adherence to the protocol of the host trial.. Secondary outcomes are level of adherence, number of dropouts, and time to drop out from the host trial.

Keywords

Patient Compliance; Randomized Controlled Trial; Reminder Systems; Study within a Trial (SWAT); Treatment Adherence and Compliance. 
The All-Ireland Hub for Trials Methodology Research, in collaboration with the Medical 22 Research Council Network of Hubs in the United Kingdom, have developed the Study Within 23 A Trial (SWAT) program, to provide studies that would investigate the effects of different 24 methods of designing, conducting, following-up, analyzing, and interpreting evaluations of 25 health care, within clinical trials.(1-3)

Explanatory trials where the focus is on measuring the efficacy of an intervention in ideal conditions, consider adherence to the trial intervention as an integral part of the trial 28 methodology, and accordingly strict treatment fidelity monitoring measures are put in 29 place.(4) Conversely, pragmatic clinical trials seek to measure the effectiveness of an 30 intervention in routine clinical practice environments, and more often than not, adherence to 31 the intervention being evaluated is not considered. $(5,6)$ Therefore, adherence in pragmatic 32 clinical trials, like the host trial in this SWAT, presents a challenge. $(7,8)$

The World Health Organization (WHO) defines adherence as the extent to which a trial 34 participant's behavior corresponds with the trial protocol in terms of taking medications as 35 prescribed, attending clinical appointments, and/or executing lifestyle modification 36 interventions as required. $(9,10)$ Non-adherence has been well recognized for years to be a 37 common issue that significantly impacts clinical outcomes and health care costs.(11-13) Poor 38 adherence is particularly challenging in cardiovascular trials, which mostly aim to manage risk 39 factors and improve cardiovascular disease prevention. $(12,14)$ While accepting that routine clinical cardiovascular secondary prevention practice also suffers from low adherence rates, yet reduced adherence in cardiovascular clinical trials can have a negative effect on the trial sample size and estimation of the treatment effects. $(15,16)$ 
(NARC),(12) the collection of non-adherence data varies substantially among cardiovascular

of the calculated efficacy of the study intervention. $(12,17)$ Hence, clinical trials may not

This study is a SWAT. The host trial is evaluating the effectiveness of an intensive lifestyle modification program in controlling risk factors and preventing stroke and cardiac events in

A great deal of effort is often expended in recruiting participants to randomized trials.(18) 59 Following the challenge of recruiting the required number of participants, there is the problem of ensuring that all participants remain in the trial and adhere to the trial intervention as required. $(7,12)$ Non-adherence to the trial intervention has serious implications, resulting in decreasing the statistical power of the study, impacting negatively on the trial outcomes and increasing the risk of attrition bias due to incomplete data. $(15,16,19)$ In addition to the loss of valuable knowledge, low adherence rates can result in research resource wasting and 
A distinction is made between intentional and unintentional non-adherence.(19, 21) 67

Unintentional non-adherence is a passive process whereby patients fail to adhere to 68 prescribing instructions through forgetfulness, carelessness, or circumstances out of their 69 control such as health literacy or cognitive impairment.(19, 21) In contrast, intentional non- 70 adherence is an active decision on the part of patients, which may be based on perceptions of 71 symptom reduction, fear of side-effects, fear of addiction, or perceived inefficiency of 72

$\begin{array}{ll}\text { treatment.(22, 23) } & 73\end{array}$

The issue of non-adherence is particularly problematic in cardiac rehabilitation (CR) trials. 74 Both intentional and unintentional non-adherence were reported in secondary prevention for 75 cardiovascular disease.(12, 19, 24) Evidence showed that approximately $31 \%$ of patients 76 reported unintentional non-adherence, while 9\% reported intentional non-adherence.(22) 77 Despite the proven benefits of $C R,(25,26)$ eligible patients do not always agree to take part in 78 CR. Of those patients that do agree to participate, many do not adhere to the CR programs as 79 recommended.(10, 12) A recent meta-analysis that included almost 400,000 patients, 80 estimated that adherence to secondary prevention of cardiovascular disease is only $57 \% .(27) \quad 81$ Similarly, an evaluation of lifestyle changes among cardiovascular patients in five European 82 countries indicated that only $50 \%$ of patients modified their lifestyles in accordance with 83 recommendations. $(10,28) \quad 84$ Furthermore, there is evidence that only $50 \%$ of patients adhere to cardioprotective 85 medications 1 year after commencing treatment. Of those taking the medications, about 50\% 86 follow the treatment sufficiently to gain a therapeutic benefit.(10, 29) This is similar to the 87 estimated prevalence of poor adherence to cardiovascular prevention and medications as $\quad 88$ reported by WHO.(30) 
A Cochrane systematic review evaluating the effectiveness of methods and strategies to 90 promote patients' adherence in CR programs(17) demonstrated that there is a need to devise 91 strategies to improve adherence in such programs and evaluate their effectiveness.(17) 92 Telephone reminders to non-responders were effective in increasing recruitment to trials.(18) 93 As yet, this strategy has not been tested to improve adherence to trial interventions. 94 Telephone reminder intervention could have a greater effect on non-intentional non- 95 adherence in CR trials. This SWAT aims to assess the effectiveness of telephone reminders on 96 participants' adherence within the cardiovascular host trial.

Participants who have been recruited and randomized to the intervention arm in the host randomized control trial will be further randomized for this SWAT. Patients in the intervention arm of the host trial will attend a 16-week multidisciplinary lifestyle program, which includes healthy lifestyle changes such as smoking cessation, healthy food choices, increasing physical activity levels, and management of dyslipidemia, diabetes, and hypertension. The intervention program of the host trial program will consist of 16 sessions of 2.5 hours each per week. Each of the weekly sessions will incorporate an individualized meeting between a multidisciplinary healthcare team (which includes a physiotherapist, dietitian, nurse, and physician) and each patient. The multidisciplinary team will review the progress of each patient and health goals. The weekly sessions will also include a one-hour group exercise program and an educational workshop.

Participants allocated to the intervention arm of this SWAT will receive telephone call 
the participant is reminded of their appointment date and time (appendix 1). There will be 16

appointments (one appointment every week) for the lifestyle intervention program in the host

trial. Therefore, the SWAT participants will receive a telephone call reminder every week over

the 16-week of lifestyle intervention program. A telephone call reminder will be received two

business days before each appointment. Up to three calls will be made if the line was busy or

there was no answer. For confidentiality reasons, no messages will be left on voicemail.

Comparator

Participants allocated to the control group in this SWAT will not receive any telephone

reminders. At baseline assessment, patients will be given a schedule of their visits throughout

the intervention period. These patients will have no telephone call reminders before their

appointments.

Method for allocating to intervention or comparator

Patients will be allocated to the telephone reminder intervention or to control group via

sealed randomization envelopes, in an equal ratio of 1:1. The investigator will not be able to

identify which arm each patient will be allocated until the sealed envelope has been opened.

128

The randomization scheme will be produced using the PROC PLAN ${ }^{\circledR}$ procedure of the SAS ${ }^{\circledR}$

software package. 
- Adherence to the protocol of the host trial.

Secondary outcome

- Level of adherence to the protocol of the host trial.

- Number of dropouts from the host trial.

- Time to dropout from the host trial.

\section{Definition of outcomes}

In the context of this study, the primary outcome of adherence is defined as $100 \%$ attendance.

The secondary endpoint of level of adherence, is measured as the percentage of attendance

of all allocated visits, within the host trial.

\section{Analysis plan}

Analyses will include appropriate descriptive analyses, and between-group comparisons using

SPSS software. The primary analysis is the difference in adherence rate between those

receiving the telephone reminders and those not receiving the reminders. This will be done

using chi-square tests. Odds ratios and $95 \%$ confidence intervals will be calculated. The

secondary analysis is time to drop-out. This will be plotted by Kaplan-Meier survival curves

and using the log-rank test to compare the two randomized groups. Cox regression will be

used to adjust for age, gender, treatment allocation in the host clinical trial. Analyses will be

undertaken on an intention-to-treat basis, using two-sided statistical significance at the $5 \%$

level. Data will be presented as proportions and percentages (adherence rate) or as the 
Ethical approvals for the SWAT and the host trial and have been sought and granted; therefore, we do not anticipate any ethical issues arising. The SWAT protocol has been registered in the SWAT Repository of the Northern Ireland Network for Trials Methodology Research (SWAT number 81). However, there is currently no evidence to support the effectiveness of telephone reminders to improve adherence in a randomized trial. A priori, we cannot pre-empt that telephone reminders may have an adverse effect on adherence.

Adherence in this study is presented as a trial methodology issue. However, adherence to the intervention might also be seen as an issue for the intervention delivery. We argue that this SWAT is not designed to investigate the outcomes of the host trial intervention. The SWAT will demonstrate the effect of telephone call reminders on patient adherence rates, which could be used in clinical trials going forward. Nevertheless, if within the host trial, we do find that patients randomized to either arm of this SWAT study show improved outcomes within the intervention arm of the host trial, then we could assess if telephone reminders should be considered as part of the host intervention delivery into routine care. As such, it could have

This research was financially supported by the School of Nursing and Midwifery, the National University of Ireland Galway (Research Seed Grant Scheme 2018). We also thank our 
1. Smith V, Clarke M, Devane D, Begley C, Shorter G, Maguire L. SWAT 1: what effects do site visits by the principal investigator have on recruitment in a multicentre randomized trial? Journal of Evidence-Based Medicine. 2013;6(3):136-7.

2. Smith V, Clarke M, Devane D, Begley C, Shorter G, Maguire L. SWAT 1: what effects do 180 site visits by the principal investigator have on recruitment in a multicentre randomized trial? Journal of Evidence-Based Medicine. 2013;6(3):136-7.

3. Treweek $\mathrm{S}, \mathrm{Bevan} \mathrm{S}, \mathrm{Bower} \mathrm{P}$, et al. Trial forge guidance 1: what is a study within a trial (SWAT)? Trials. 2018;19(1):139.

4. Thorpe $\mathrm{KE}$, Zwarenstein $\mathrm{M}$, Oxman $\mathrm{AD}$, et al. A pragmatic-explanatory continuum indicator summary (PRECIS): a tool to help trial designers. Journal of Clinical Epidemiology. 2009;62(5):464-75.

5. Treweek S, Zwarenstein M. Making trials matter: pragmatic and explanatory trials and the problem of applicability. Trials. 2009;10(1):37.

6. Dal-Ré $R$, Janiaud $P$, loannidis JP. Real-world evidence: How pragmatic are randomized controlled trials labeled as pragmatic? BMC Medicine. 2018;16(1):49.

7. Matsui D. Strategies to measure and improve patient adherence in clinical trials.

8. Haynes R, Yao X, Degani A, Kripalani S, Garg A, McDonald H. Interventions to enhance medication adherence.[update of Cochrane Database Syst Rev. 2002;(2): CD000011; PMID:

9. Sabaté E. Adherence to long-term therapies: evidence for action, World Health 
10. Hevey D. Adherence to Health Recommendations. In: Perk J, Gohlke H, Hellemans I,

Sellier $\mathrm{P}$, Mathes $\mathrm{P}$, Monpère $\mathrm{C}$, et al., editors. Cardiovascular Prevention and Rehabilitation.

11. Rasmussen JN, Chong A, Alter DA. Relationship between adherence to evidence-based

pharmacotherapy and long-term mortality after acute myocardial infarction. JAMA.

203 2007;297(2):177-86.

12. Valgimigli M, Garcia Garcia HM, Vrijens B, et al. Standardized classification and 205 framework for reporting, interpreting, and analysing medication non-adherence in 206 cardiovascular clinical trials: a consensus report from the Non-adherence Academic Research

13. Ferdinand KC, Senatore FF, Clayton-Jeter $\mathrm{H}$, et al. Improving medication adherence in

14. Chowdhury R, Khan H, Heydon E, et al. Adherence to cardiovascular therapy: a metaanalysis of prevalence and clinical consequences. European Heart Journal. 2013;34(38):2940-

8.

15. Hewitt CE, Kumaravel B, Dumville JC, Torgerson DJ. Assessing the impact of attrition in

16. Adamson J, Hewitt CE, Torgerson DJ. Producing better evidence on how to improve randomised controlled trials. BMJ. 2015;351:h4923.

17. Davies $\mathrm{P}$, Taylor F, Beswick A, et al. Promoting patient uptake and adherence in cardiac 
18. Treweek S, Pitkethly $M$, Cook J, et al. Strategies to improve recruitment to

randomised trials. Cochrane Database Syst Re. 2018, Issue2. Art. No.: MR000013. DOI:

10.1002/14651858.MR000013.pub6.

19. Lehane $E$, McCarthy $G$. Intentional and unintentional medication non-adherence: a

comprehensive framework for clinical research and practice? A discussion paper. International

20. Bower P, Brueton V, Gamble C, et al. Interventions to improve recruitment and

retention in clinical trials: a survey and workshop to assess current practice and future

228 priorities. Trials. 2014;15(1):399.

21. Lehane $\mathrm{E}, \mathrm{McCarthy} \mathrm{G}$. An examination of the intentional and unintentional aspects of

22. Lowry KP, Dudley TK, Oddone EZ, Bosworth HB. Intentional and unintentional

23. Gadkari AS, McHorney CA. Unintentional non-adherence to chronic prescription medications: how unintentional is it really? BMC Health Services Research. 2012;12(1):98.

24. Cooper A, Jackson G, Weinman J, Horne R. Factors associated with cardiac rehabilitation attendance: a systematic review of the literature. Clinical Rehabilitation. 2002;16(5):541-52.

25. Giannuzzi P, Saner H, Björnstad H, et al. Secondary prevention through cardiac 
26. Dalal HM, Doherty P, Taylor RS. Cardiac rehabilitation. BMJ. 2015;351:h5000.

27. Naderi SH, Bestwick JP, Wald DS. Adherence to drugs that prevent cardiovascular 246

disease: meta-analysis on 376,162 patients. The American Journal of Medicine. 247 2012;125(9):882-7. e1.

28. Shepherd J, Alcalde V, Béfort P-A, et al. International comparison of awareness and 249 attitudes towards coronary risk factor reduction: the HELP study. Journal of Cardiovascular 250 Risk. 1997;4(5-6):373-84.

29. Ockene IS, Hayman LL, Pasternak RC, Schron E, Dunbar-Jacob J. Task force\# 4252 adherence issues and behavior changes: achieving a long-term solution. Journal of the 253 American College of Cardiology. 2002;40(4):630-40.

30. World Health Organization. Adherence to Longterm Therapies: Evidence for Action. 255 Geneva: World Health Organization; 2003. 


\section{Appendix 1}

Reminder Call Script:

This is [the hospital name/health network name/and study name] at [ the department name],

calling to remind you about an appointment for [patient's name] on [day and date] at [time] 263

at [the cardiac rehabilitation center name]. Please arrive 15 minutes prior to your 264

appointment time to allow the registration process. If you have any questions, do not hesitate 265

to contact the study investigators on [phone number]. We look forward to welcoming you. 266

Thank you. 